19

\title{
Commerce Information System Based on the WWW
}

Meiqi Fang, Jinglei Li, Bin Yin

Information School, Renmin University Of China, Beijing, P.R.China.

Tel: 086-10-62513256. Fax: 086-10-62513254.

Email: mqfang@public.bta.net.cn

\begin{abstract}
With the emergence and popularity of Internet and WWW, information sharing and exchange have become global, and Electronic Commerce has been considered widely. Commerce Information System (CIS) is an information system whose goal is to process commerce transactions. We will discuss the two-level concept model of CIS in this paper-- In macro sense, it is EC, while in micro sense, it is Commerce Oriented Information System(COIS). EC, consisting of many independent COIS, is more complicated and huge. Being an internal information system in the EC environment, COIS aims to carry on trade and gain more benefits, so it differs in many aspects from the traditional MIS in an organization. Compared with traditional MIS, COIS is expert in processing and utilizing
\end{abstract}


external data to coordinate with the EC environment. We will also discuss the differences in system goal, function, architecture and design. To develop such a complicated information system as COIS on Internet. Such method as 00 and Components is very useful. In later part of this paper, a model of this two-level Commerce Information System using such methods will be demonstrated.

\author{
Keywords \\ EC, COIS, SIM-EC, O0,WWW.
}

\title{
1. INTRODUCTION
}

Since 1960s, modern computer technology has been applied to Economy and Management extensively for the inventory management, order processing, salary management, etc. In the traditional sense, the Management Information System (MIS) in an organization focuses on managing mainly internal data and assisting managers for decision making. It often has an unified goal and an integrated architecture.

With the development of human society, information processing is becoming more and more industrialized, socialized and global. The scope of information sharing and exchange is extended day by day, and the type of information becomes more complex and varied. Under these circumstances, a large number of complicated external information of an company is playing a more important role, so the MIS in the traditional sense can not adapt to it. Thus the concept of Information System(IS) becomes wider. A system in which information is communicated in a trade, even in the whole society, is a more complicated and huge IS than the traditional MIS in an organization.

A Commerce Information System(CIS) is an information system whose goal is to process commerce transactions using modern Information Technology(IT).

Electronic Commerce, which has started in USA and Canada, means using computers and networks to carry on trade among the businesses and consumers that collectively make up what we call commerce. Thus, as an information system, EC system can be seen as a macro CIS.

In order to develop smoothly and coordinately, EC must have two basic parts: infrastructures and commerce regulations. Infrastructures means communication network and devices including IT. Commerce regulations are the rules by which every company must abide if it want to do business in EC. Fundamentally, the Internet is an infrastructure which can connect any computers anywhere on the planet with higher speed and lower costs. And EDIFACT is a kind of commerce regulations.

In the EC which changes frequently, every company's IS is an independent unit. It is able to control, manage and develop itself for its own benefit and adapting to the EC environment. This kind of IS also aimed at commerce 
transactions, so it can be seen as a micro CIS; we call it Commerce Oriented Information System(COIS). These COISes are integral parts of EC. They have high adaptability and are expert in processing and utilizing external information, then it has gone much further than traditional MIS in an organization.

In this paper, We will discuss two concepts-in micro sense and in macro sense--of Commerce Information System. Then we will analyze the new changes of Commerce Information System in micro sense based on the Internet and WWW in the aspects of system goal, and their function, architecture and design. After that we will discuss some protocols and techniques that can support the Commerce Information System based on WWW. At the end of the paper, a model of this twolevel Commerce Information System will be given. We would like to share our experience with you.

\section{THE TWO-LEVEL CONCEPTUAL MODEL OF COMMERCE INFORMATION SYSTEM}

In 1980 s, people began to transfer standard messages through computer network and named it Electronic Data Interchange(EDI). EDI originated from the transportation industry, then extended to the commerce, administration, medical treatment, etc. The emergence and popularity of the Internet and WWW enabled the information exchange via network in a global environment, which propelled EDI application in commerce greatly and motivated Electronic Commerce also.

EC has started in USA and Canada in 1990s. It is a new way of doing business. In brief, EC means to trading electronically. In the Internet environment, EC not just only refers to doing business on the net, but also involves all the commerce activities such as problems solving, costs reduction, values adding, and searching for new business opportunities. It consists of a series of commerce activities on the network including inquiring, purchasing, exhibition, ordering, producing, storing, transportation, electronic payment, etc.

With the wide use of IT (Information Technology) in international trade and business, it has become a trend to make commerce internationalized by using computer tools, network and communication techniques.

EC is developed in order to be adapted to the commerce trend of taking the world as a whole market. EC increases and develops very fast and enables almost all the companies on the Web to connect with other entities more closely and easily, meet their customers' demands more quickly, and select the proper partners in a wider area.

In general, ways to share and exchange information in EC system have three levels as follows:

A. Each company sends E-mails on Internet to exchange information. In this way information has only a single type--E-mail, and the form of exchange is also simple. 
B. Each company has its own Web homepage on WWW to deliver its products information, advertisements and news. At the same time it can also visit the homepage of the other companies on the Web. Although the types of information exchanged in this way become more diverse, the information is still as static as lists of products. There is no timely information exchanges, and the companies only receive information passively, not interactively.

C. The exchange between companies is more interactive. On the one hand companies release product information. through its homepage on the WWW; on the other hand they take part in a series of information exchange activities through the Web. These activities may contain inquiring, quoting, ordering, Customs declaration, payment through Electronic Fund Transfer (EFT), etc. And besides, they go beyond the boundary of an organization, use external information to do business on the network and improve their management in order to increase their benefits. In addition to response to the information from outside, the internal IS of the companies must be able to understand and identify useful information, and then adjust and improve the rules of response. In this way, the types of information become more complex and broad. The forms of exchange are more varied. And the processing ability is also strengthened.

The traditional Information Management enables to organize and manipulate the predefined data. When business is conducted and information is exchanged on the Internet; data to be treated are not all predefined, stored on the internal databases, some of them which may be structured or unstructured are accessed externally through the Internet. This is an entirely new scenario which requires newer approach than the traditional Information Management. The now popular Knowledge Management (KM) offers one of the approaches. What is going on over the Internet is all dynamics. The strong point of the $\mathrm{KM}$ is its ability to deal with dynamics. Knowledge Management is commonly defined as an attempt to put processes in place that can capture and reuse an organization's knowledge so it can be used to generate revenues. Here knowledge should be construed as both internal and external knowledge of an organization. In contrast with Information Management, Knowledge Management enables to dynamically link structured and unstructured information with the changing rules by which people apply it. The technologies needed to achieve KM are: groupware, workflow, document management, intranets and extranets, decision support tools, etc. Information management tools such as intranets, publish and subscribe, data warehouse, OLAP., and others can be used too. Data Mining could be relabeled as Knowledge Management, since they are technologically alike.

The scenario envisioned in the above $C$ represents the new height to be reached in the development of the EC. Perhaps delving a little into the subject is in order. Commerce Information System described in the above $\mathrm{C}$ has two meanings.

In the macro sense, the Commerce Information System is such a system like 
this: Corporations or companies manage their internal information, and at the same time they participate in the exchange of information on the Net. For example, they can release product information and search for new commerce opportunities through the WWW. By exchanging information on the Net they compare the prices and conditions to select the best partner. Such organizations as the banks, transportation companies, tax departments, and the Customs use the Net and EDI system to exchange necessary documents by signing contracts. Then the goods are transported by the transportation companies and the buyers pay for them through the EFT. Each organization involved in the whole trade process is a subsystem connected by the Internet. The whole system is a Commerce Information System in the macro sense, since its aim is to trade electronically.

In the micro sense, the internal IS (the information system in a company) must be adjusted to keep up with the trend that the commerce activities have gradually become global. It is different from the traditional MIS. That is, this kind of internal IS in the EC system should, in addition to the conventional functions of an IS, aim at participating in the business on the Net and improving its adaptability, interaction, and security; and in particular it should have the capability of extracting, processing, and assimilating the external information. In this sense, when the internal IS has the capability of participating in the EC activities, it has become a Commerce Information System in the micro sense;, we will call it a Commerce Oriented Information System(COIS).

The relationship between the EC system and the COIS is shown in figure 1 .

The two levels of the Commerce Information System interact with each other and can't be isolated. Without the smoothly running of each COIS, the EC can not develop coordinately. Conversely if the EC is not developed coordinately and smoothly, no COIS can obtain its own benefit.

Firstly, the COIS is the basic cell of the EC system and undertakes part of the trade processing. The Internet particularly the WWW. is the medium of communication. Some Information Service Centers (See fig. 1) also exist in the EC system.

Secondly, each COIS has close connections with others. These subsystems affect and adapt to each other, coexisting in competition and cooperation.

Thirdly, some new assemblages may arise dedicated to the system-wide information services. Some of them assume responsibility of securely transferring all kinds of standardized documents and keep track of the whole transfer process to provide for evidences for the arbitration that may arise in the future. And some collect and arrange commerce information to provide services such as the information retrieval by various companies in the system, and the market condition and quotations required by the traders. Others serve for the payment through the EFT..

Finally, small scale companies can not get enough profit when they operate one COIS to compete with other big ones. The small companies could pool into one COIS like an exhibition operating cooperatively on the network . 


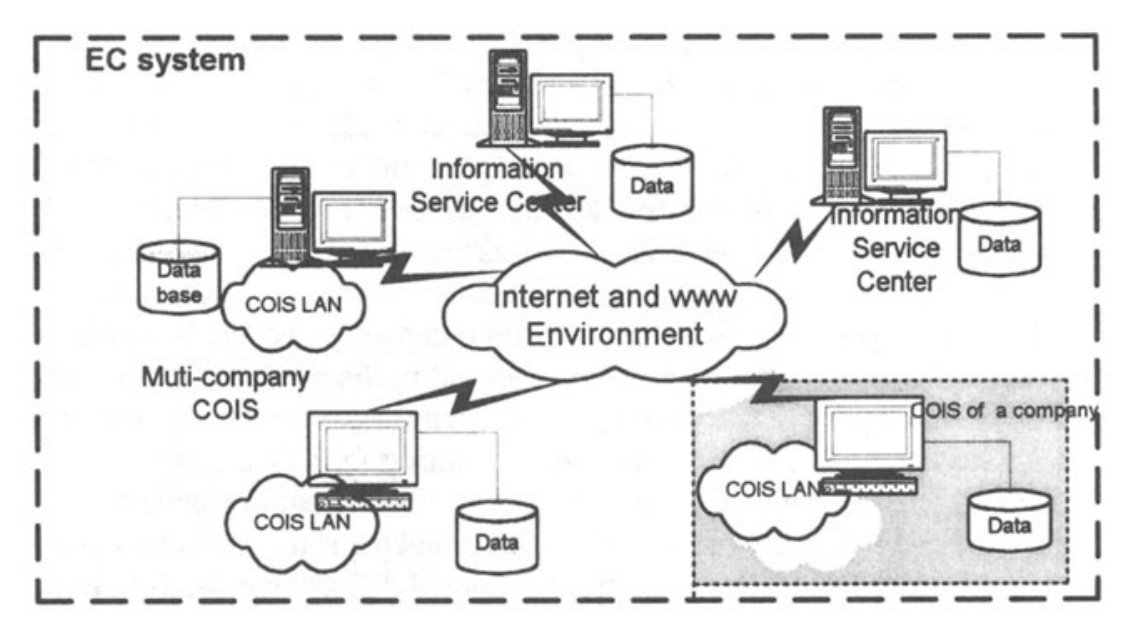

Figure 1.Two-level concept model of IS.

Here the EC system means the whole system which consists of mono-company COIS, multi-company COIS and the Information Service Center.

Multi-company COIS is a more complicated COIS which serves many small companies. somehow it is like an exhibition.

\section{MIS VS. COIS}

\subsection{Goal}

The goal of traditional MIS is to improve the management of a company by using the modern Information Technology (IT). As a system which connects with its environment not so closely, the MIS aims at organizing the internal information effectively and providing the information to the managers to help them to make decision.

In addition to the aims mentioned above, the COIS can also collect and assimilate a great deal of external information, then process these information to support decision. It also searches for partners, sends and receives messages. For instance, a manufacturer in the EC system sends out a request of searching for certain kinds of merchandise. After receiving the answer from the Information Service Center, it analyzes and processes the information, and make some appraisals such as the demand, the average price, etc. Then it will adjust the structure of products to increase profit. Compared with the MIS, it has become the main goal of COIS to participate in the electronic trade and utilize both external and internal information, not just using internal information.

\subsection{Function}


The functions of the MIS include collection, store, process, transmission and provision of information. More than these, the COIS also have such functions as reception, selection, analysis of, and response to external information (e.g. messages), and, in addition, adjustment of response rules according to the environment.

\subsection{Logical Architecture}

The architecture of the MIS usually has two layers: a central database and many application programs including user interface and transaction rules, interlinked by a network. In respect of coordinate computing model, Framework-based model will be adopted if security and timing are more critical and if funds permit. For example, data system of banks or ticket selling system of airline companies can use this model. A common company prefers to $\mathrm{C} / \mathrm{S}$ computing model or Net/Fileserver computing model for the limited funds. But what is distributed is process, not data (Physically, the system is still composed of a central database and some programs). Now there are many DB products supporting distributed DB in which data are stored in different places, however, the data compose an integral DB logically.(See fig. 2)

COIS has three layers in logic: Data Service, Application Service, Business Service. Data service is in charge of DB management and keeping the security and integrity of data ( This layer is done by DBMS provided by DB producer generally). Application Service contains the following process: providing user interface, receiving requests from users and sending requests to DB server for data querying. In addition, it can also be accessed and queried by other WWW users. In the Business Service layer there are some components which are necessary for electronic trade, external information exchange and internal information management. These components are used for the purposes of security, trade rules, information filters, information selecting, etc.(See fig. 3) 


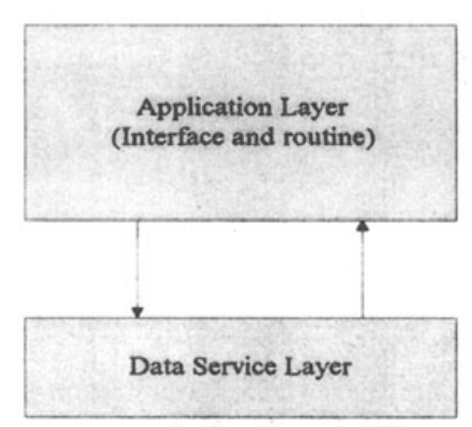

Figure 2 Traditional MIS Two-Layer logical Architecture.

Figure 2 Traditional MIS Two-Layer logical Architecture.

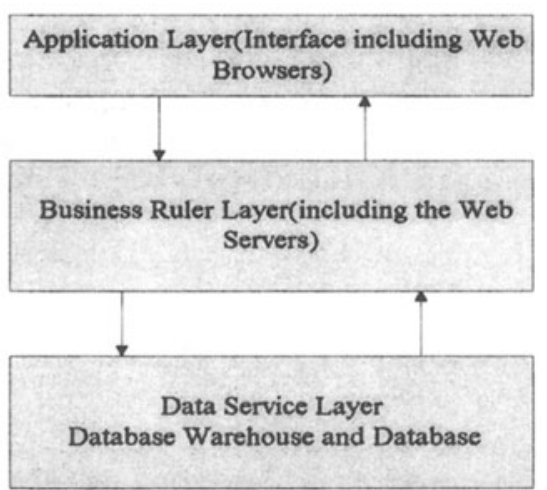

Figure 3 COIS Three- Layer logical Architecture

Figure 3 COIS Three- Layer logical Architecture.

\subsection{Design Methodology}

Analyzing and designing MIS mainly follows the structured method and other traditional methods. Sometimes people use the Life Cycle Method, going through four stages-analysis, design, implementation and maintenance-step by step. Sometimes they use the Prototype Method in which a basic framework is established firstly, then improved while being tested.

Since the adaptability of the COIS is more essential, and the COIS is an open system which interacts with the environment closely through WWW and also has more interoperability; it is not enough to focus only on analyzing relationships of internal information when we design a COIS. We should think how to design the system so as to enable it to adapt to the new environment quickly at the lowest cost even when the environment (software/hardware platform) changes frequently. Specifically, there are two points as follows:

- It is appropriate to adopt three logical layers model. Variable information like business rules can be made to be the pluggable components stored in middle layer. What needs to do in the application layer and the data layer is to integrate these pluggable components. By this way the adaptability and interoperability of the COIS will be increased.

- Since the environment becomes more variable and complicated as the time goes on, and the users' requirements thus harder to ascertain, the Prototype Method design can not adapt to the changing conditions any more. New method must be worked out. A new idea based on the CAS theory is more suitable for developing the complicated IS in complicated and varied 
environment. (See Reference [4])

\section{TECHNIQUE FOR EC DEVELOPMENT}

In the EC system exists a great number of spatially distributed partners which depend on as well as oppose each other in terms of the benefits. The system manages many different types of business transactions on heterogeneous hardware and software. Experience shows the security and audit of EC system are much more important than those in common systems. When disputes arise between two partners, arbitration is needed. The requirements of EC push the development of WWW. On the other hand the development of WWW is the best foundation of the EC. In this section we will give a brief description of the development technique of the WWW and share our experience of our sample EC with you.

The $\mathrm{OO}$ methodology is a very natural and efficient method to learn about the outside world. The $\mathrm{OO}$ supports programming, system design and even system analysis. Similar to other application system, the COIS should consist of the interface objects, the operation objects and the basic objects. the interface object is the interface between the users and the COIS. Many successful design of system interfaces provided good samples for our design. According to the commerce requirements we can design user friendly interface. the basic objets provide for the common function on every application. They can all be reused..

The operation objects reflect the business rules and the system controls; they should be designed on the basis of careful analysis of the domain under consideration. Later in an example to be discussed in this paper there is a document class and object which consists of such attributes as data elements, data segments, etc., and such operations as edit, copy, modify, send and receive... Many commerce documents are its subclass, like the quotation document, the payment document, etc.... These classes are useful in practice.

The software components are very useful for the COIS too. Many software publishers have made a substantial number of software components, for example, OCX and ActiveX from Microsoft. To buy suitable components can speed up the development of the COIS. There are some protocols for heterogeneous components which can communicate with and call each other in heterogeneous hardware and software environment. These protocols are Object Management Group's (OMG) Common Object Request Broker Architecture (CORBA) and Microsoft's Distributed Common Object Model (DCOM), and etc. Each of them provides different plumbing for the components to communicate with each other; but neither of them is a complete solution for network programming. Quite a few of them are needed for a complete solution.

Under. CORBA scheme an Object Request Broker acts as an intermediary (broker) between the heterogeneous components. The components, though unable to talk to each other because of heterogeneity, can talk to ORB through the common language IDL (Interface Definition Language). A Client component's 
request for services, written in IDL, is mapped into the host language (i.e., $\mathrm{C}$ or $\mathrm{C}++)$ of the server.

CORBA can be used in cross-platform environments. An ORB exists for every popular operating system, and for some operating systems. there are two or four different vendors' ORBs. Native implementations of CORBA are available on just about every platform. For example, Digital's Object Broker is on 20 platforms including Mac Os, AIX, MVS, OS/2 Warp, OS/400, Digital Unix, openVMS, HPUX, Windows 3.x, Windows 95, and Windows NT. All these just show how crossplatform CORBA is.

DCOM can be used for Windows-only systems But now Microsoft has turned management of the DCOM standard over to the Open Group. It is expected that DCOM will be in multiplatform environment too. DCOM is released for Windows 95 and NT. German giant Software AG and Digital (now bought by Compaq) are porting DCOM to many non-Windows platforms.

In COM as well as DCOM, objects communicate with each other through "interface", which is defined in the OLE design specification as "a grouping of a set of semantically related functions into a named unit." Interfaces are defined in $\mathrm{C}++$ as virtual classes.

Under DCOM a request for services starts with a call for an object proxy on the client side. The proxy passes object calls over the network to a stub on the server side. A Proxy is a piece of code that is responsible for putting interface parameters into a message buffer and passing that buffer to a channel (a named pipe or a RPC channel or a network), which is responsible for transferring the data to another process. A Stub is a piece of code that retrieves data for a channel, unpacks the parameters from the buffer, and calls the client code to use the parameters. The Object proxy consists of a Proxy Manager and one or more interface proxies. (See ref. 1)

Notice the ways components communicate with each other under CORBA and DCOM are different: Communications are done through the intermediary of ORB under CORBA; while no such intermediary exists under DCOM, because COM and hence DCOM is not dependent on any particular language implementation (See ref.1) and is used in the Windows-only environment. In the case of CORBA, however, there was a problem which had not solved until 1994. ORB is supposed to be a "universal translator" who can speak to anybody speaking any language (in fact, only those programming languages to which. IDL is mapped to). But before 1994, there was no standard way for one vendor's ORB to talk to other's. In 1994, the OMG ratified the Internet Interoperable ORB Protocol (IIOP). With this specification, objects created with one vendor's development tools are able to talk to objects created with another vendor's. In other words, the ORBs have a standard protocol for talking to each other. (See ref. 2)

Java is very useful to the EC development too. There are many JavaBeans and Applets. We could buy them or download them from the Internet.

Based on RSA and other theories there are many different encryption, 
electronic signature methods. For the security of fund transfer many security protocols were set up based on the cooperation of many companies. For example Security Sock Lever(SSL) Security Hyper Text Transfer Protocol, SecurityMIME, Security Electronic Transaction(SET) etc. It is important that we should know the different usage of these protocols so that we can use them correctly. S_HTTP and SSL can be used on Web business. S-MIME can be used on E-mail. SET supports credit card transaction..

\section{A INFORMATION SYSTEM MODEL IN THE WWW ENVIRONMENT - SIM-EC}

\subsection{Function and structure of the SIM-EC}

The SIM-EC (Simulate Electronic Commerce) software is an on going project. This software is used to simulate the EC environment and all the active individuals in that environment. The EC environment is based on the Internet where new technologies have been widely applied in the commerce transactions, especially in the WWW. It is proposed to show how the various businesses in the SIM-EC strengthen their ability to handle the outside relations as well as enhance their ability of the internal-management by adapting their information system to the rapid change of the outside information environment. The SIM-EC consists of many distributed LANs(Locate Area Network) that connect with each other through the Internet. The system is composed of various units (programs) that simulate all kinds of organizations in the market economy. These units are divided into two types. One is the automatic role, including Manager, Mailer, Bank, Customs and Consumer, whose activity is done entirely by our program; The other is the business role, including the Recycler, Manufacturer, Wholesaler, Retailer and Transporter. The business role is the program simulating a company's business activity in the EC environment. The user acting for the company interact with others by way of this IS. The relationships of these business roles are cooperative and competitive. The programs of these roles are running on the diverse computer hardware and software platforms, and reside in different locations. All kinds of business roles and automatic roles constitute an open Electronic Commerce environment. SIM-EC roles and their relationships can be seen in figure 4 :

In this environment, the EC information system is different from the traditional MIS in the following aspects.

- Linking the information system of an organization with the outside is a necessity and is going to play a more and more decisive role.

- The emergence of the new information industry, such as information service industry and information management industry, the electronilization of the management, and the socialization of the informilization. In the SIM-EC, 
many automatic roles are the embodiment of these new industries and they constitute the macro environment of the SIM-EC.

- The IS of an organization should be more or less scalable and extensible. An organization, whether big or small. should continuously adjust the process rule in the modules and the relationships between the modules in the IS. This kind of IS is represented by many application programs which are run interactively the users in the SIM-EC.

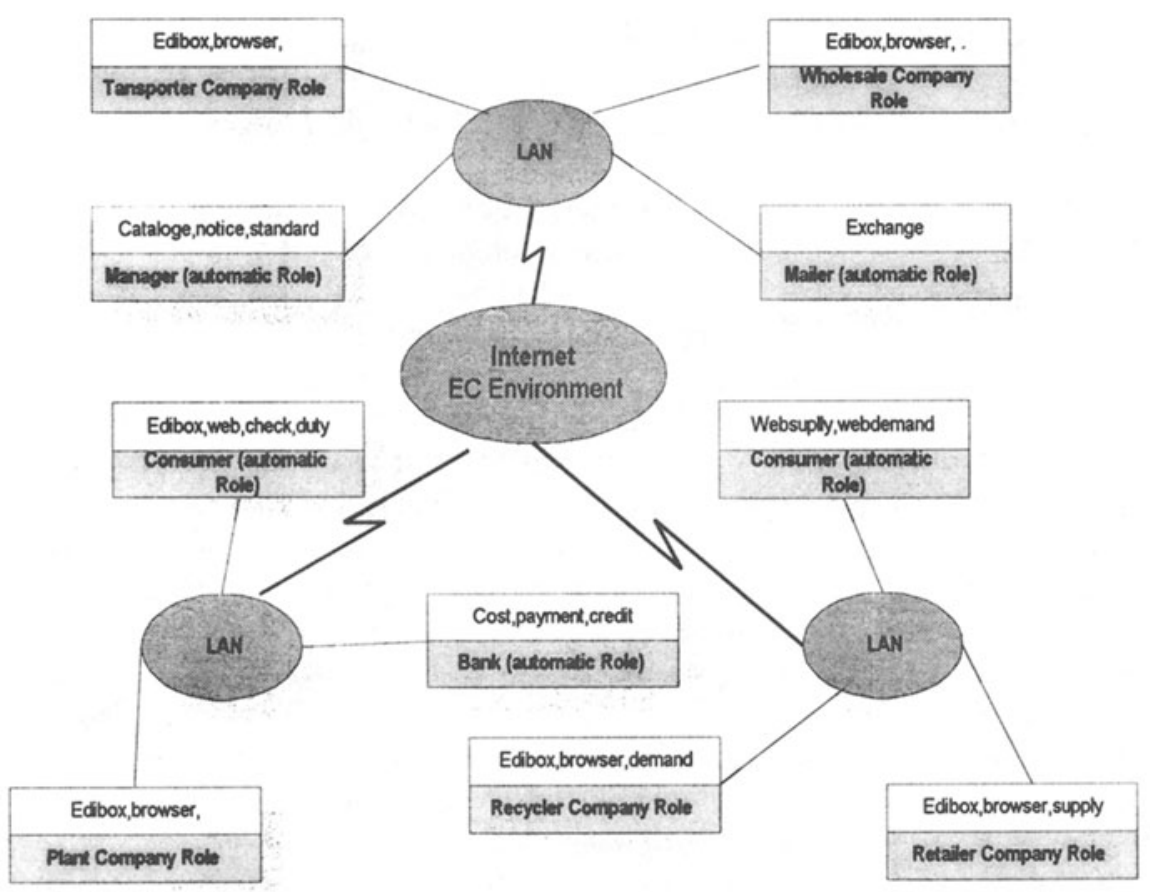

Figure 4 SIM-EC Roles and Their Relationships.

Legend:

\begin{tabular}{|c|}
\hline Interface \\
\hline Role \\
\hline
\end{tabular}

\subsection{1 the automatic role in SIM-EC}

In the real EC environment, there should be some organizations which take care of 
the data communication, data security and data management, and some government departments as well(See ref. 7). In the SIM-EC we make a few additions; The details are as follows:

Mailer: act as server for data communication and security.

Manager: decide and announce some commerce activity rules, provide services of arbitration and front-end Web application like product catalog;

Bank: offer credit authentication for business operations, and provide online shopping with verification and payments by the EFT.

Customs: undertake the government's function as goods inspection and import/export control.

Consumer: simulate consumer and labor market so that the business roles can sell their products and buy the necessities for production, then the cycle of production, distribution, exchange and consumption can be set up and the companies can keep running.

Government departments, such the Customs Service and Commodity Inspection, work as the market regulators, and they have relatively advanced IS too. Their goal is to keep fair competition and to enforce the national policy. The way to implement the goal efficiently is through the digital electronilization of the management. For example, in the import and export business, an enterprise must make the Customs declaration. In the SIM-EC, automatic the role of Customs automatically carries out the task of inspection. It stores the records of all the declaration and commodity inspection and has all the regulatory procedures programmed. So the Customs can trace the flow of certain material, trace a company's import/export activities, and it will find out whether the company has broken the law after comprehensively analyzing these activity records.

\subsubsection{The COIS in the SIM-EC}

In this EC environment, it is critical for a company to communicate with its environment. A careful designed interface is helpful for a company to achieve the finial goal-profit. In the SIM-EC, the business role is designed to realize the interface function between the company itself and the outside EC environment. This IS serving the business role is what we call "COIS" above.

What can COIS do?

- Continuously release the information of their products by means of the EDI and the home pages on the Web.

- Up-to-the-minute respond to an inquiry of price or a demand for further contact from the clients, and calculate the cost, profits, and estimate delivery date according to the reasonable regulation.

- After the conclusion of a bargain, update the consumer, inventory and account information, and notify their partners of any changes that may require them to take some action in cooperation with the others. 
- Collect market information, such as the product price, demand and trend, in timely manner

- keep track of the clients and flow of product.

- Continually adjust forecast model and commerce rule.

The company manager is responsible for making decision on the purchase, manufacture and sale on the basis of the COIS's information.

In the real world, the company is faced with disperse separate data and diverse function commonly existing in the Internet environment. So the way of building the COIS is through the integration as follows:

- Devices integration, that is the integration of the different hardware (computer and network devices) and system software.

- Data integration: that is synthetically using technologies such as data warehouse, OLTP and OLAP to describe the relationship among these disperse data. Here some application programs are needed to handle these data. In the SIM-EC these distributed data are stored in different network and computer. Some data can be gotten by preprocessing some documents and the information exchanged with other sources such as downloading the notice from the Manager role or subscribing to some special information.

- Function integration: that is providing interface between the humans and the machine, and describing the business flow and analyzing the data to support management and decision from the various approaches. This function integration is related to the BPR(Business Process Rebuild - what is popular in company management at present) and has two goals: one is to improve service quality and expand market; the other is to reduce cost and control profit. In this course, the process unit should be the work flow. Since every company is just a segment in the industry link, it is sufficient to have a simplified model which only controls the input, output and process.

The above discussion of the COIS is in fact a part of what constitutes the entirety of the COIS in the CIS model, but discussed in the context of the SIM-EC.

\subsubsection{The information exchange in the SIM-EC}

There are three types of information exchange among COISes, including business-business, business-consumer and business-government. In the past, if two companies need to exchange information between their MIS, they would first sign a contract, establish a steady relationship and define a rigid communication standard, then they can exchange data between their MIS through EDI. At present, in the SIM-EC, there are many methods to exchange information among the COISes, such as the EDI, the Web, and the publish and subscribe.

In the case of one company using the Web, it can get information by browsing 
the Web pages and send information by launching a Web site. Using the publish and subscribe method, one company's COIS can automatically send special information to the other COIS that have subscribed the request, and in the same way, this COIS can also receive message from the others.

Now, to different type of transaction and in different phase of a certain transaction, companies can select proper approaches or even combine them to receive, respond, select and analyze information. In the SIM-EC, a company will inquire the Bank about the credit status of its partners and clients, or inquire the Customs about its goods situation. In this case, it is convenient for the company to directly login on the Web server of the Bank or the Customs to interchange information. However, when big trade company process their transactions., it is more reasonable to use the EDI, because of the enormous and constant communication affairs.

In the SIM-EC, the Manager is an important role. It can classify and refine the message, provide product catalog service, fulfill the subscription information of companies, and so on. The Manager represents the newly emerging industry of information service and information agents. All the company roles need to interact with Manager role.

In the SIM-EC, there is a Consumer role which simulates the consumer market; it has its own mode of consumption and perception of external market. It leads to different market tactics in this game. The mode and the perception are all adjustable. The adjustment can be done by experiment or can be self-adjusting according to the macro economic model. All these are to collect the data for the purpose of analyzing them later to determine whether the running model of market economy will undergo some essential changes in the EC environment.

\subsection{The design and implementation idea of the SIM-EC}

\subsubsection{Adopting the idea of dividing layers}

The essential idea of the SIM-EC's design and implementation is to divide layers. Dividing layers, in essence, is to simplify the actual thing. The idea of dividing layers has a profound influence in the development of computer hardware and software. After dividing layers, the explorer can concentrate on only a certain relevant layer, and then, do the detailed design and development. Between adjacent layers, only the interface and services can be seen, the service is the collection of the functions that the lower layer can provide. Thanks to this idea, software gradually evolve into system software and application software. System software evolve into operate system, database system and so on. In the network communication area, the port-port communication execute also by dividing layers. In the SIM-EC, first, the whole software program can be divided into three layers: environment, interface and individual. The environment mainly refers to the automatic roles. The interface mainly refers to the three relationships of the 
business-business, business-consumer and business-government; we handle these relationships through multi-level directory, which are stored in different computer software and hardware platform, including microcomputer running UNIX OS, PC server running NetWare $3.11 / 4.11$ or windows NT 4.0 and PC running windows $3 . x$ or win 95 . Their connection means are various. As to the individuals, each company's COIS can further be divided into three layers. They are the application layer, the business rule layer and the data layer. Three layers constitute a multi-tier client/server architecture. In the WWW environment, the Web server acts as business rule layer and the browser program as a application layer. All the layers in the SIM-EC are illustrated in figure 5

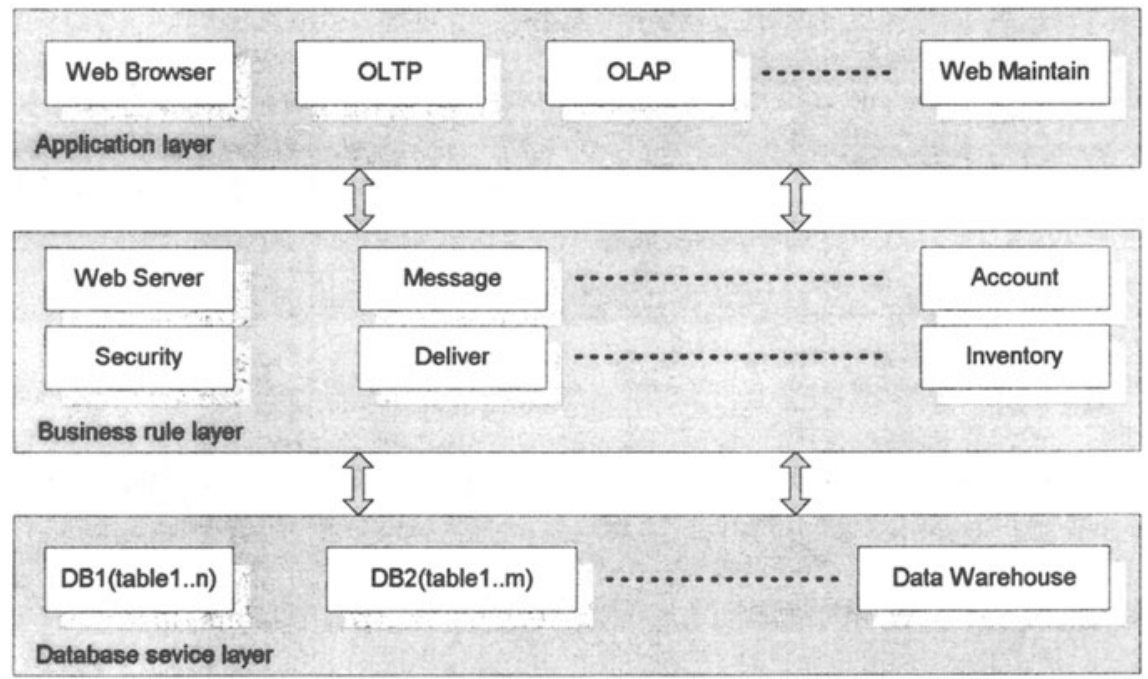

Figure 5. SIM-EC three-layer C/S architecture including some objects.

\subsubsection{Separate the Process from the Data}

The basic requirement of the layer dividing is to separate the process from the data, to improve the process independent. In a system with clearly divided layers, the field where the data affect program control flow is defined in a small scope. In the SIM-EC, it is complying with the EDIFACT(Electronic Data Interchange For Administration Commerce and Transportation) to create or open a document. The course of creating or opening is independent to document type or content, so is the course of automatic answering or abstracting document. We adopt database technology to bring about process independence. That method is to store the easily modified information of program control in the database, then executing some select sentence, the program can know what is to do next. Using the process 
independent technology, we can claim some update about document format and type, without updating the programs, among which EDI executes.

\subsubsection{Using the Object Oriented method}

We apply the OO(Object Oriented) method to all phases from analysis, design to implementation. The $\mathrm{OO}$ technology in programming language level has been discussed in the preceding part of the text. The $\mathrm{OO}$ methods in the logical level are mainly practiced in the business rule layer including the process rule. The logical model of SIM-EC also embodies the essential idea of $\mathrm{OO}$ : abstraction, encapsulation, inheritance and polymorphous. Using the $\mathrm{OO}$ method to abstract the COIS, we can find that the system is no more than some business objects and their relationships, though in COIS, the data is widely distributed in area and the functions required are varied. Consequently, we draw a conclusion that complexity is a composition of comparatively simple basic business objects.

In the SIM-EC, we classify the entirety of business into several types, which respectively deal with the material flow, currency flow and information flow. We can add each process rule to every type of business and encapsulate them to become some objects. Material flow includes the inventory object, dock object and production plan object. Currency flow is composed of the bank account object, cash account object, etc. Information flow is composed of such objects as document object, client object and so on. To say more specifically, the document object is a basic class (or superclass), and it can generate some subclasses such as $\mathrm{QD}$ (quotation document), $\mathrm{OD}$ (order document), $\mathrm{PD}$ (payment document) class and so on. These document subclasses have some common method and attribute inheriting from superclass, and respectively have their own special method and attribute. For example, all the subclass have the common method to create a new document, which is to merge every data element of the document into a string that can be encrypted and transmitted; all subclasses have the common attribute showing their record mark when they are inserted into document table. However, the QD and OD respectively have their own method and attribute. For example, the QD has a method to automatically create a corresponding PRD(price document) to send to client, and has an attribute to maintain a number of PRD. As to the OD, it has a method to call the register of client class and has an attribute to maintain a number of client.

In a word, we use the business objects to encapsulate all the process description of these businesses in the SIM-EC.

\subsubsection{The Prototype and The Component Ware}

We employ the prototype and component method in the SIM-EC design and implementation. From a long-term aspect, once a COIS has been setup, it will be constantly modified even rebuilt for the purpose of running efficiently. The reason 
lies in that the business processes will alter with the change of outside environment. So, if the COIS itself wants to have that adjusting and perfecting ability, it is necessary to use two methods in design and implementation prototype and component.

First, as discussed in the three layer model above, we should divide the business objects in comparatively abstractive degree and higher level of the granularity. These business objects and the relationships among them reflect the real world, and they constitute the frame of the COIS. After establishing a COIS frame with an excellent structure, we have only two things left. One is to constantly enrich the process ability of every single business object in the system. The other is to describe complex relationships among these objects in a deeper and deeper degree. In the last section, we introduce the dividing objects of SIM-EC(the detail see figure 5), this design is a process applying the prototype and the $O O$ method.

Next, in the system implementation level, the programmer can use several program languages to build the objects and generate independent small programs, which encapsulate the single business and the operation rule on it. We integrate these small programs to create our entire software. We have a tool to describe the objects and reflect the relationships in the real world, through the composing relationships of these small programs. The way to satisfy this demand is software component. The composed small independent programs feature with good interoperability.

In the SIM-EC, some components can be bought from the software market, either needing OCXs or ORBs (depending on the OS used), but these components are mainly applicable to the application layer to provide a convenient interface between human being and machine, while some components are applicable to the data layer to manipulate database. In the SIM-EC, we also need to encapsulate business rule into components. These business components are so customized for specific fields that most of them are developed by ourselves. During this development, we can use Java, $\mathrm{C}++$, Delphi, VB. Little of these business components can be stored in the same machine as the application program. Because most of these business components are used by many machines, we concentrate these components in several certain servers so that we can manage them conveniently and shift business rule easily. For example, during the document transmission, we provide a component in the mailer server to all users, which can symmetrically encrypt and limit users to an identical encryption rule. Another example, in Bank or Customs Web server, there are Java Applet or ActiveX available for visitor login and to verify visitor. That Applet or ActiveX can be developed with Java or VB programming language

A few words at the conclusion, the component method provide a convenient and effective way to the implementation of SIM-EC system, and is helpful to application of prototype. 


\section{SOME ISSUES FACED BY THE EC}

Nowadays many virtual shops have been successfully established on the WWW. But the EC system discussed in this paper contains not only business-to-consumer trade like the virtual shops, but also business-to-business and business-togovernment. Many international organizations and countries especially the western ones attach great importance to EC. A series of standards and guides have been worked out. But as a newly emerged IS, there must be some problems during the development of the EC. For example, how does the government administrate it? How do standard trade process and law framework come to be global?

several important problems of technology are listed as follows:

\subsection{Security}

It contains three parts: legal identification, information reliability of the transfer, protecting Web site and the Intranet from the intrusion by the illegal users. So far some techniques and methods have been proposed, such as SET protocol, Firewall, Public/Private Key encryption algorithm, etc. But these are not enough for real electronic commerce on the network.

\subsection{Bandwidth}

Besides improving the network equipment, we should study new transfer protocols to accelerate response and save bandwidth. Without the popularity of high bandwidth, the development of the EC will be restricted.

\subsection{The problem of complexity and openness}

A computing environment inside an company has been considerably complicated. When we connect the Intranet with the Internet, there must be some serious challenges on performance, security and openness of an internal IS. How the whole system in the EC environment can achieve steady and coordinated development is a complicated problem demanding further discussion.

\section{CONCLUSION}

We have given a brief description on the growing EC and COIS. The need of the EC is urgent. The WWW and the EC interact with each other and mutually push forward their own development.

In this paper, we have introduced some concepts and the structure of the EC and COIS. Then we discussed some relevant techniques. Finally we showed a sample of the EC simulation environment we are working on. Our main conclusions are: 
A. From logical point of view, the traditional MIS is an integration of interestoriented subjects, aiming at efficient business management. MIS structure is more simple compared with EC. EC structure is much more complex, because it consists of many different subjects which have distinct interests.. There are more levels on EC. Besides the aspect of technique, there are some more essential aspects on EC, such as security, certification, arbitration, etc.

B. Normally, the EC system should include several different hardware and software platforms. We need to develop techniques to support the communication and use different applications and software modules. Many protocols, like CORBA, DCOM and IIOP, and many standards, like EDIFACT and X12 are very useful for these demands. The applications developed by Java could run on heterogeneous hardware and software. Java is very useful tools for the EC development.

C. The $\mathrm{OO}$ analysis, design and programming is suitable for describing the reality. It is very useful for the EC development. Components software are very suitable for EC too.

D. Based on the basic principle of the Complex Adapted System theory and considering the IS should be adapted to the outside world, this new method improved prototyping methodology. It is more suitable for development of EC.

E. Due to EC development, many companies which provide new information service are emerging and exploring fast. It is significant for the progress of human society. Every individual could take the advantage of this opportunity to make more contribution to the society, at the same time he could get more reward from society.

\section{REFERENCES}

Toohey \& Toupin, Building OCXs, pp. 33-5

Special Report on Bridging Networked Applications, BYTE, April 1997 pp. 93-105

Chen Yunian \& Chen Yu. Information System Engineering, Science Press, Beijing, 1990.

Jim Carr, "E-Commerce applications", INFOWORLD, June 23,1997,.

Michael Jay Tucke "EDI and the Net a profitable partnering" Datamation, April 1997,.

Sean Doolittle, "Securing the Cybermarket - the future of Electronic Commerce on the Internet", PC Today, June 1997.

"Electronic Commerce and the NII DRAFT FOR PUBLIC COMMENT", http://iitfact.nist.gov:94/doc/Electronic_Commerce.html 


\section{BIOGRAPHY}

Mei Qi Fang is a Professor of Computer Information Systems at Renmin(People's) University, Beijing, China. She is the B.S. of mathematics, Tsinghua University of China, and the M.S. of computer science, Renmin University of China. Two books among the five she authored or co-authored, are Decision Support Systems and Application, published by Naing Jing University, and Computer Simulation, published by Renmin University. She wrote over 30 articles that appeared in journals in China and the US. She had over ten of computer industry experience where she directed or participated in major software development projects for Chinese state owned enterprises before becoming an educator. Her research interests include management information systems, computer aided education, software, computer simulation and e-commerce.

Jinglei $\mathrm{Li}$, and Bin Yin are currently postgraduates at Information School of Renmin University, Beijing, China. They are both the major developer of SIM_EC. Their interests also include: management information systems, ecommerce, complexity adaptive system and computer simulation on economics. 